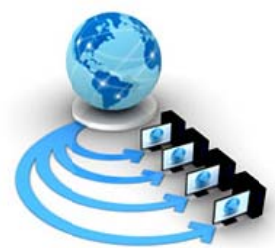

International Journal of Advanced Research in Computer Science

REVIEW ARTICLE

\author{
Available Online at www.ijarcs.info
}

\title{
FAST UNSUPERVISED BAYESIAN IMAGE SEGMENTATION WITH ADAPTIVE SPATIAL REGULARIZATION: A REVIEW
}

\author{
Manisha \\ M.Tech Scholar \\ ECE Department, D.C.R.U.S.T, \\ Murthal,India
}

\author{
Gitanjali Pandove \\ Associate Professor \\ ECE Department, D.C.R.U.S.T, \\ Murthal,India
}

\begin{abstract}
Image segmentation is a process of dividing the image in to some distinct regions. These region shave specially coherent in nature and have similar attributes. This technique is widely used for image analyses and to interpret the desired feature. In this present paper we will study about the hidden Markov random fields and find its expectation maximization algorithm.
\end{abstract}

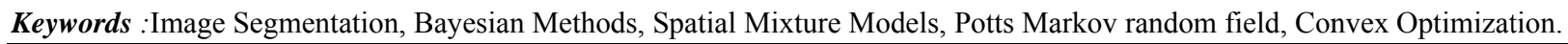

\section{INTRODUCTION}

If we study about the image possessing system, the resulted image may contain some irregularities or defects that may affect our process. Furthermore these kind of defects can be adjusted by various kind of techniques like increase the number $\mathrm{f}$ picture from the same scene which decrease the effect of defect and by using a higher quality instruments, but such methods which are based on the external hardware are consume more time and they increase the cost too [1]. So to avoid such effect of external hardware we often used computer programs which consume very less time and reduce the cost. For example to remove the noise defect we can use smooth filter which effectively reduce the noise content and filter the image or to change the contrast level in a low contrast image we can use image histogram by which level can be scaled. Such correction of various defect in image is called image pre processing [2]. After removing the defects the process of image segmentation occurs. For example segmentation of food image which means to distinguish automatically the food products from an image is obviously carried out after image acquisition because this process of segmentation is completely carried out by the computer programs and there is no need of human intervention between the process the computer itself recognize the food items. If we defining the image segmentation in simple words, the image segmentation process divide the image in to several well defined regions. All these regions have similar pixels characteristics and attributes.As image segmentation is a important task because all the object classification and object measurement i.e. interpretation task is completely based on the results of segmentation process. A high quality of effort are being used to obtain an optimal segmentation techniques but till now there is no such technique are available. [3]. But still there are various kind of segmentation techniques are available which gives effective results. In food industry four kind of segmentation techniques are most used which are thresholding-based, region-based, gradient-based, and classification-based segmentation. But these techniques cannot provide a high accuracy result if it used for a wide range of food products. Some further methods which are ancombined effect of above techniques are also being developed that compromise on accuracy of results [4].

The complete research paper is described in five sections. The introduction is described in Section I,Section II describes literature review, Section III describes problem formulation,Performance parameter describe in section IV, Finally, Section V describes the conclusion of paper.

\section{LITERATURE REVIEW}

This section will provide the brief description and highlights the contribution, remarks and factors of the work done by the researchers. Many attempts have been made in the past to achieve the maximum accuracy while segmented the images.

Pereyra, Marcelo et.al highlighted about Bayesian image segmentation methodology that is used to compare the simulation results by an estimated result which is generated by an algorithmic method which is Markov chain Monte Carlo algorithm. Accuracy was found out 95\% 99\%.Regularization parameterforLung, Bacteria, Brain and SAR was0.065, 0.110, 0.095, and 6.6314 respectively [1].FitsumMesadi et.al highlighted about Disjunctive Normal Level Set method. On the other hand another technique DNSM was also used for single object segmentation. DICE Score was achieved up to $98.5 \%$ This technique was provided better computational time [2].ErtuncErdil et.al proposed about segmentation using nonparametric joint shape. Feature priors technique was used. Dice score and average hausdorff desistance was achieved up to 0.9409 and 10.5742. after calculating all these calculation we can calculate a average Dice score on basis of ground truth which is 0.9153 [3]. Shifeng Wang et.al proposed MRF model. MRF model is suitable for expressing a connection between the random variables. The system had the four sensors forward-looking LRF (LRF1), Accelerometer (Acc), camera (Cam), Downward looking (LRF2). Classification accuracy forMRF, acceleration based, Image based and downward LRF was 97.5\%, 69.4 $\%, 88.8 \%$ and $82.3 \%$ respectively [4].Ronghua Shang et.al described about Fast FCM clustering based on key pixels. The algorithm was separated the whole image into two parts 
Key pixels,non-key pixels. For Image $256 \times 256,1$ look SA, 2 look SA, 4 look SA were 97.05, 98.48 and 98.65 [5].Dona Francis et.al described third generation of artificial neural networks Pulse Coupled Neural network was used. The PCNN neuron consists of three compartments receive field, modulation and pulse Generator.Total 40 images are input to PCNN engine and false positive rate is 0.031989 [6]. AnuvaKulkarni et.al proposedthatRW algorithm had been used for image segmentation. Hash codes were used instead of raw pixels and hamming distance between the hash codes as the distance metric. Ground truth value Image1, Image 2, Image 3 and Image 4 was96.61 \%,96.49\%, 88.92 \%and98.29 \%respectively [7].Javier Gimenez et.al highlighted about Bayesian model, Potts Model are also used for comparison MSE, Standard Deviation and Mean of Estimators computed over 100 Realizations of a Potts Model.For several values of $\beta$ Gaussian datawith $\sigma=$ $15, \mathrm{k}=1[8]$.

TABLE: 1Literature Review Table

\begin{tabular}{|c|c|c|c|c|}
\hline Reference & Paper Title & $\begin{array}{l}\text { Research Methodology } \\
\text { used }\end{array}$ & Major Findings & Research prospects \\
\hline$[1]$ & $\begin{array}{lr}\text { Fast unsupervised } \\
\text { Bayesian } & \text { image } \\
\text { segmentation } & \text { with } \\
\text { adaptive } & \text { spatial } \\
\text { regularization } & \end{array}$ & $\begin{array}{l}\text { Bayesian image } \\
\text { segmentation } \\
\text { methodology is used } \\
\text { \&compare the } \\
\text { results with the estimates } \\
\text { produced by the Markov } \\
\text { chain Monte Carlo } \\
\text { algorithm }\end{array}$ & $\begin{array}{l}\text { Accuracy: } \\
95 \%-99 \% \text {. } \\
\text { Regularization } \\
\text { parameter? } \\
\text { For Lung, } \\
\text { ? = } 0.065 \text {; } \\
\text { For Bacteria, } \\
\text { ? }=0.110 \\
\text { For Brain } \\
\text { ? }=0.095 \\
\text { For SAR, } \\
\text { ? }=6.6314 .\end{array}$ & $\begin{array}{l}\text { This technique is } \\
\text { applicable for the } \\
\text { number of images } K \\
\text { (is unknown). These } \\
\text { applications are used } \\
\text { to } \\
\text { Ultra sound and PET } \\
\text { image segmentation }\end{array}$ \\
\hline$[2]$ & $\begin{array}{l}\text { Disjunctive Normal } \\
\text { Parametric Level set } \\
\text { with application to } \\
\text { image Segmentation }\end{array}$ & $\begin{array}{l}\text { Disjunctive } \\
\text { Level Set method is } \\
\text { used. On the other hand } \\
\text { another technique } \\
\text { DNSM has recently been } \\
\text { used for a single object } \\
\text { segmentation }\end{array}$ & $\begin{array}{l}\text { DICE Score : } \\
98.5 \% \\
\text { The technique } \\
\text { provides better } \\
\text { computational } \\
\text { time. }\end{array}$ & $\begin{array}{l}\text { The proposed } \\
\text { DNLS-multiphase } \\
\text { approach has the } \\
\text { highly desired } \\
\text { properties that it is } \\
\text { less sensitive to } \\
\text { initialization, and its } \\
\text { computational } \\
\text { cost and memory } \\
\text { requirement }\end{array}$ \\
\hline$[3]$ & $\begin{array}{l}\text { Nonparametric Joint } \\
\text { Shape and Feature } \\
\text { Priors for } \\
\text { Image Segmentation }\end{array}$ & $\begin{array}{l}\text { MNIST handwritten } \\
\text { digits data set is used. } \\
\text { Segmentation using } \\
\text { nonparametric joint } \\
\text { shape and feature priors } \\
\text { technique is used. }\end{array}$ & $\begin{array}{l}\text { Dice score : } \\
0.9409 \\
\text { Average } \\
\text { Hausdorff } \\
\text { distance } \\
10.5742 \\
\text { The average Dice } \\
\text { score (Hausdorff } \\
\text { distance) results } \\
\text { on all test images } \\
\text { with respect to } \\
\text { ground } \\
\text { Truths are } 0.9153 \\
(1.7899) .\end{array}$ & $\begin{array}{l}\text { The proposed } \\
\text { method minimizes } \\
\text { an energy function } \\
\text { that includes a joint } \\
\text { nonparametric shape } \\
\text { and feature } \\
\text {. }\end{array}$ \\
\hline [4] & $\begin{array}{l}\text { Two-Stage Road } \\
\text { Terrain Identification } \\
\text { Approach for Land } \\
\text { Vehicles } \\
\text { Using Feature-Based } \\
\text { and Markov Random } \\
\text { Field Algorithm }\end{array}$ & $\begin{array}{l}\text { MRF model is suited to } \\
\text { express causal } \\
\text { relationships between } \\
\text { random variables which } \\
\text { stem from the four } \\
\text { sensors } \\
\text { 1. forward-looking LRF } \\
\text { (LRF1) } \\
\text { 2. Accelerometer (Acc) } \\
\text { 3. Camera (Cam) } \\
\text { 4. Downward looking }\end{array}$ & $\begin{array}{l}\text { Classification } \\
\text { accuracy } \\
\text { MRF : } 97.5 \% \\
\text { Acceleration } \\
\text { based : } 69.4 \% \\
\text { Image Based : } \\
88.8 \% \\
\text { Downward LRF: } \\
82.3 \% \text {. }\end{array}$ & $\begin{array}{l}\text { MRF algorithm was } \\
\text { presented to improve } \\
\text { the terrain } \\
\text { classification } \\
\text { accuracy for land } \\
\text { vehicles. }\end{array}$ \\
\hline
\end{tabular}




\begin{tabular}{|c|c|c|c|c|}
\hline & & (LRF2). & & \\
\hline [5] & $\begin{array}{l}\text { A Fast Algorithm for } \\
\text { SAR Image } \\
\text { Segmentation } \\
\text { Based on Key Pixels }\end{array}$ & $\begin{array}{l}\text { Fast FCM clustering } \\
\text { based on key pixels. The } \\
\text { algorithm separates the } \\
\text { whole image into two } \\
\text { parts } \\
\text { Key pixels } \\
\text { Non-key pixels. }\end{array}$ & 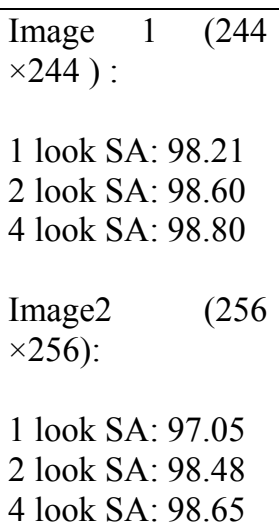 & $\begin{array}{l}\text { Improving the } \\
\text { algorithm in terms of } \\
\text { preserving edges and } \\
\text { other details of the } \\
\text { original image, and } \\
\text { the level-set methods } \\
\text { may offer a potential } \\
\text { way forward. }\end{array}$ \\
\hline [6] & $\begin{array}{l}\text { Fundus /Mage Vessel } \\
\text { Segmentation } \\
\text { Using PCNN Model }\end{array}$ & $\begin{array}{l}\text { Third generation of } \\
\text { artificial neural networks } \\
\text { Pulse Coupled Neural } \\
\text { network is used. The } \\
\text { PCNN neuron consist } \\
\text { ofthree compartments } \\
\text { 1. Receive field } \\
\text { 2. Modulation } \\
\text { 3. PulseGenerator }\end{array}$ & $\begin{array}{l}\text { Total } 40 \text { images is } \\
\text { input to PCNN } \\
\text { engine } \\
\text { FPR : } 0.031989\end{array}$ & $\begin{array}{l}\text { The algorithm's } \\
\text { characteristics of } \\
\text { pixel } \\
\text { misclassification are } \\
\text { low while comparing } \\
\text { with other } \\
\text { algorithms. }\end{array}$ \\
\hline [7] & $\begin{array}{l}\text { Unsupervised Image } \\
\text { Segmentation Using } \\
\text { Comparative } \\
\text { Reasoning and } \\
\text { Random Walks }\end{array}$ & $\begin{array}{l}\text { RW algorithm has been } \\
\text { used for image } \\
\text { segmentation } \\
\text { Hash codes used instead } \\
\text { of raw pixels and } \\
\text { hamming distance } \\
\text { between the hash codes } \\
\text { as the distance metric }\end{array}$ & 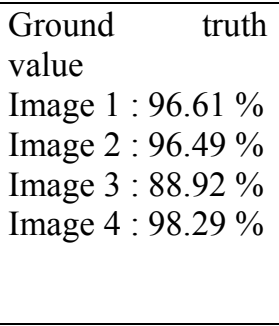 & $\begin{array}{l}\text { The algorithm saves } \\
\text { in computation time. } \\
\text { It was found that } \\
\text { Proposed method } \\
\text { performed better } \\
\text { than state of the art } \\
\text { methods. }\end{array}$ \\
\hline [8] & $\begin{array}{l}\text { When Data Do Not } \\
\text { Bring Information: A } \\
\text { Case Study } \\
\text { in Markov Random } \\
\text { Fields Estimation }\end{array}$ & $\begin{array}{l}\text { A Bayesian model is } \\
\text { used } \\
\text { Potts Model is also used } \\
\text { for comparison }\end{array}$ & $\begin{array}{l}\text { MSE, Standard } \\
\text { Deviation and } \\
\text { Mean } \\
\text { Estimators } \\
\text { computed } \\
\text { over100 } \\
\text { Realizations Of A } \\
\text { Potts Model For } \\
\text { Several Values of } \\
\beta \\
\text { Gaussian Data } \\
\text { with } \sigma=15, \mathrm{k}=1\end{array}$ & $\begin{array}{l}\text { Posterior PML have } \\
\text { interesting statistical } \\
\text { properties such as } \\
\text { consistency, } \\
\text { asymptotic } \\
\text { normality, as the } \\
\text { prior estimators do. } \\
\text {. }\end{array}$ \\
\hline
\end{tabular}

\section{PROBLEM FORMULATION}

Owing to the defects that are present in an image acquisition system, some techniques are performed to remove such kind of defects like image pre processing and histogram manipulation are performed to eliminate the noise content and to change the contrast level in an image of low contrast level [8].Later on, image segmentation process is used to distinguish the eatable items from the background by analyzing the regions. In account of this as the image segmentation process is still not well defined so not a single methods described above can perform ideally and cannot give a high accuracy results. To get the better results and to increase the efficiency we can use the combination of several techniques that may give an efficient result and also increase the segmentation speed. The main objective of the paper is to study and analyze image segmentation
Approaches [7-10]. The approaches weresuch as Markov random field and its expectation-maximization algorithm will be studied in depth.

\section{PERFORMANCE PARAMETERS}

The performance of segmentation is measure withJacard score, Precision rate, Recall rate and Dice score.

\section{Jacard Score}

For an every segmented region there exist an binary map for that which is well followed by the probabilistic algorithms and an another factor $\mathrm{T}$ which is consensus truth and from these elements we can collect Dice score [11]:

$$
\text { Dice }(\mathrm{P}, \mathrm{T})=\frac{\left|P_{1} \cap T_{1}\right|}{\left(\left|P_{1}+\right| T_{1}||\right) / 2}
$$

Here $\mathrm{nis}$ a AND logical operator which used for an definite function, $j$ describes the size of an set (i.e., the number of 
voxels belonging to it), and $\mathrm{P} 1$ and $\mathrm{T} 1$ shows the set of voxels where $\mathrm{P}=1$ and $\mathrm{T}=1$. Dice score element normalize the true positives numbers to the distinct segmented are i.e. it have similar identity like the F score which is an harmonic mean of an recall curve and if desire we can also transform it into theJacard score.

\section{Recall Rate}

Recall Rate measure how many of the positives does the model. It is the proportion of positive cases that were correctly identified, as calculated as [12]

\section{Precision Rate}

$$
\text { Recall }=\frac{A}{A+B}
$$

Precision Rate is how many of the returned documents are correct. $\mathrm{P}$ is the proportion of the predicted positive cases that were correct \& calculate as [13]

\section{Segmentation Time}

$$
\text { Precesion }=\frac{A}{A+C}
$$

It is the total time takes place during segmentation of an image.

\section{Accuracy}

The accuracy of a test is completely based on the capability of the test to distinguish the patient and healthy cases efficiently. To compute the accuracy of an system we calculate the ration of true positive and true negatives in all the performed cases. Mathematically, this can be stated as [14]:

$$
\text { Accuracy }=\frac{T P+T N}{T P+T N+F P+F N}
$$

\section{CONCLUSION}

In this paper we try to present a innovative approach for the efficient image segmentation process. Our new approach is basically relied on the Bayesian estimation method which is used for estimating the variable regions that are being hide and have irregular parameters $\beta$. The estimator thus use for the estimation of hidden region is relied on the minute variance analysis of a Bayesian model and combined with the convex relaxation.

\section{REFERENCES}

[1] Pereyra, Marcelo, and Steve McLaughlin. "Fast unsupervised Bayesian image segmentation with adaptive spatial regularization." IEEE Transactions on Image Processing 26.6 (2017): 2577-2587.

[2] Mesadi, Fitsum, Mujdat Cetin, and TolgaTasdizen. "Disjunctive Normal Parametric Level Set With Application to Image Segmentation." IEEE Transactions on Image Processing 26.6 (2017): 2618-2631.

[3] Erdil, Ertunc, et al. "Nonparametric Joint Shape and Feature Priors for Image Segmentation." IEEE Transactions on Image Processing (2017).

[4] Shifeng Wang et.al "Two-Stage Road Terrain Identification Approach for Land Vehicles Using Feature-Based and Markov Random Field Algorithm" IEEE Intelligent Systems, vol 10, issue 99 ,pp 1-8, June 2017.

[5] Ronghua Shang et.al "A Fast Algorithm for SAR Image Segmentation Based on Key Pixels" IEEEJournal Of Selected Topics in Applied Earth Observations and Remote Sensing, Issue 99 , pp 1-17, Oct 2017.

[6] Francis, Dona, and Jemima Jebaseeli. "Fundus image vessel segmentation using PCNN model." Green Engineering and Technologies (IC-GET), 2016 Online International Conference on. IEEE, 2016.

[7] AnuvaKulkarni et.al "Unsupervised Image Segmentation Using Comparative Reasoning and Random Walks" IEEE Global Conference on Signal and Information Processing (Global SIP), 338 -342, 2015

[8] J. Gimenez, A. C. Frery, and A. G. Flesia, "When data do not bring information: A case study in Markov random fields estimation," IEEE J. Sel. Topics Appl. Earth Observ. Remote Sens., vol. 8, no. 1, pp. 195-203, Jan. 2015.

[9] J. Gimenez, A. C. Frery, and A. G. Flesia, "When data do not bring information: A case study in Markov random fields estimation," IEEE J. Sel. Topics Appl. Earth Observ. Remote Sens., vol. 8, no. 1, pp. 195-203, Jan. 2015.

[10] Y. Boykov and V. Kolmogorov, "An experimental comparison of mincut/max-flow algorithms for energy minimization in vision," IEEE Trans. Pattern Anal. Mach. Intell., vol. 26, no. 9, pp. 1124-1137, Sep. 2004.

[11] X. Cai, "Variational image segmentation model coupled with image restoration achievements," Pattern Recognit., vol. 48, no. 6, pp. 2029-2042, Jun. 2015.

[12] Guangpu Shao*, JunbinGao†, Tianjiang Wang*, Fang Liu*, YuchengShu*and Yong Yang"Image Segmentation Based on Spatially Coherent Gaussian Mixture Model'IEEETransactionsonNeural Networks and Learning Systems, vol. 24, no. 2, Feb. 2014.

[13] Jai Puneet Singh _, NizarBouguila "Spatially Constrained Non-Gaussian Mixture Model for Image Segmentation" IEEE 30th Canadian Conference on Electrical and Computer Engineering, pp 172-177, 2017.

[14] Juanfen Sun, ZexuanJi "Bounded Asymmetric Gaussian Mixture Model with Spatial Constraint for Image Segmentation"Proc. IEEE Conf. ComputerVision and Pattern Recognition, pp. 373-377, 2016. 